\title{
OPERATIONAL STATUS AND FUTURE UPGRADES OF BEPC
}

\author{
Y. Z. Wu for the BEPC team \\ Institute of High Energy Physics, P.O. Box 918, Beijing 100039, China
}

\begin{abstract}
In this paper the operational status of Beijing ElectronPositron Collider(BEPC) in recent years is described. The luminosity upgrades have been performed in recent years. By lowering the vertical $\beta$ function at interaction point the peak luminosity is nearly doubled. The single interaction point in BEPC is also studied experimentally. The results show that beam-beam tune shifts decrease significantly compare to the two IP's operation. The overall performance of dedicated synchrotron radiation operation is improved by installing new insertion device and optimising the beam lifetime. The future upgrades of BEPC are briefly introduced. The design studies of a new $\tau$-charm factory has been carried out continuously. On the other hand the further upgrade potential based on existing machine is being exploited.
\end{abstract}

\section{INTRODUCTION}

Beijing Electron-Positron Collider(BEPC) has been in operation for 10 years, working for high energy physics experiments as well as synchrotron radiation research in dedicated or parasitic mode. The running time is about 5500 hours/year, in which roughly $40 \%$ for particle physics, $30 \%$ for dedicated synchrotron radiation and $30 \%$ for others(machine study, etc.).

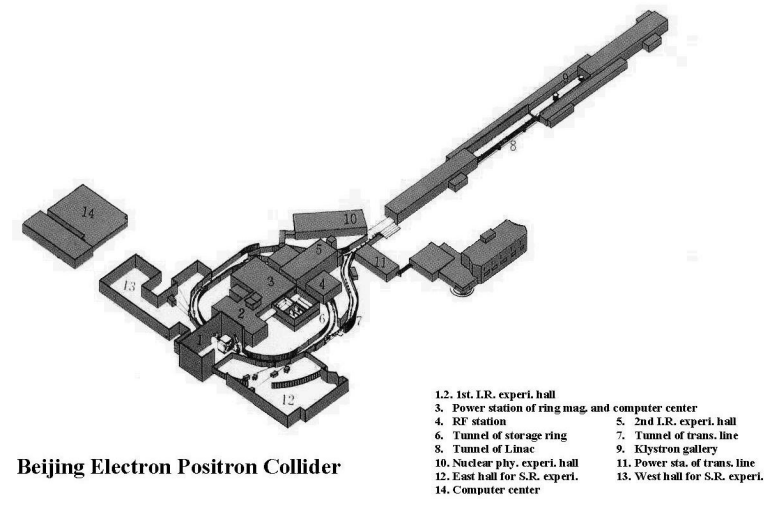

Figure 1: The schematic of BEPC
BEPC as a collider mainly works in the $\tau$-charm energy region for physics experiments. Since 1989 BES(Beijing Electromagnetic Spectrometer) detector has accumulated

- 10 million $\mathrm{J} / \psi$ events

- 3.7 million $\psi^{\prime}$ events

- $22.3 \mathrm{pb}^{-1}$ integrated luminosity at $\mathrm{D}_{\mathrm{s}}$, etc.

BEPC also serves as the unique $\mathrm{x}$-ray synchrotron light source in China known as Beijing Synchrotron Radiation Facility(BSRF). There are eight beamlines(five from 2 wiggler magnets, three from bending magnets) and 12 experimental stations. More than four hundreds users have been utilizing BSRF as the tools in different scientific research programs.

In the past several years the upgrade program is carried out mainly aiming at increasing the luminosity of BEPC. Major items include:

- linac energy upgrade(from $1.3 \mathrm{GeV}$ to $1.55 \mathrm{GeV}$ )

- improvements for control and beam diagnostic system

- luminosity upgrade for BEPC collider ring

- BES and BSRF upgrades

Up to now the basic goals of above upgrades have been achieved. The overall efficiency (in terms of integrated hadron events/day) of BES physics run is doubled. The performance of dedicated synchrotron radiation operation is also substantially improved.

\section{OPERATIONAL STATUS}

\subsection{BEPC present operation parameters}

The BEPC facility has been decribed in detail in several documents[1,2,3]. The accelerator facility is located in IHEP, Beijing. A 200 meter long linac provides electron and positron beams with the energy up to $1.55 \mathrm{GeV}$. The storage ring with a circumference of $240.4 \mathrm{~m}$ is capable of operating up to $2.8 \mathrm{GeV}$. However, because of the strong interest in $\mathrm{J} / \psi$ physics in recent years, since 1996 performance has been optimized at $1.55 \mathrm{GeV}$ beam energy mainly. In the spring of 1999 the physics experiment is measuring $R$ value. The $R$ value scan experiment works at the beam energy region from $1.0 \mathrm{GeV}$ to $2.5 \mathrm{GeV}$. This is a somewhat difficult task for machine because sometimes it works at lower energy than the design. The beam energy of dedicated synchrotron radiation operation is $2.2 \mathrm{GeV}$ since 1990 to provide soft $\mathrm{x}$-ray and VUV photon beams. It is hoped that the operating energy is to be increased to 
$2.5 \mathrm{GeV}$ or higher. A list of the principal operating characteristics of BEPC may be found in Table 1.

\begin{tabular}{|c|c|c|}
\hline & $\begin{array}{c}\text { Colliding } \\
\text { beam mode }\end{array}$ & $\begin{array}{c}\text { Dedicated } \\
\text { S.R. mode }\end{array}$ \\
\hline Beam energy $(\mathrm{GeV})$ & $1.0 \sim 2.8$ & 2.2 \\
\hline Inj. Energy(GeV) & $1.0 \sim 1.55$ & 1.3 \\
\hline Bunch/beam & 1 & $50 \sim 80$ \\
\hline Emittance(nm.rad) & $400(1.55 \mathrm{GeV})$ & 79 \\
\hline Tunes & $5.81 / 6.78$ & $8.72 / 4.80$ \\
\hline$\beta$-functions at IP & $1.2 / 0.05$ & $18 / 18$ \\
\hline RF frequency(MHz) & 199.536 & 199.536 \\
\hline Total current(mA) & $45(1.55 \mathrm{GeV})$ & $80 \sim 100$ \\
\hline Beam lifetime(hour) & $5 \sim 10$ & $\sim 20$ \\
\hline
\end{tabular}

Table 1: Principle parameters for current BEPC operation

\subsection{Operation with lower- $\beta$ lattice}

For the luminosity upgrades, the original proposal was to install a mini- $\beta$ insertion(compact permanent quadrupole magnets)[4]. But later it was found that the bunch length of BEPC storage ring is significantly longer than the proposed vertical $\beta$-function at $\operatorname{IP}(3.6 \mathrm{~cm})$ [5] even an attempt was made to reduce the coupling impedance by shielding most of bellows and removing 2 kickers. Therefore the proposed mini- $\beta$ lattice was modified by relocation of the insertion quadrupoles $35 \mathrm{~cm}$ closer to the interaction points. The current distance from IP to the first quadrupole is $2.15 \mathrm{~m}$. This allows the operation with lower vertical $\beta$ function, say, $5 \mathrm{~cm}$ instead of $8.5 \mathrm{~cm}$ at interaction points.

Since 1996 the extensive machine studies with lower $\beta$ configuration have been performed mainly at $1.55 \mathrm{GeV}$ beam energy which the physics program required in recent years. The peak luminosity has reached $4.9 \times 10^{30} \mathrm{~cm}^{-2} \mathrm{~s}^{-1}$. See Figure 1. The maximum vertical beam-beam parameter is about 0.033 . The hadronic event rate at $\mathrm{J} / \psi$ energy seen by BES detector in routine operation is increased by a factor over 2 .

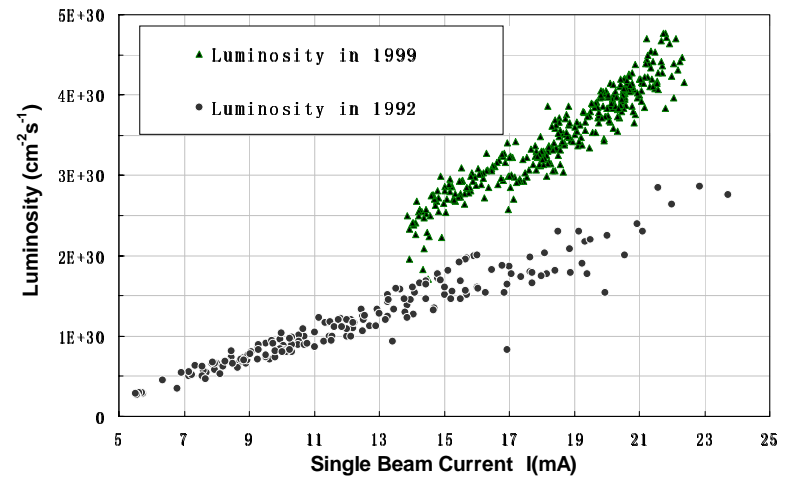

Figure 1: Luminosity increase with lower $\beta$ optics

\subsection{Single Interaction Point Experiments}

BES is the only detector for particle physics experiment on BEPC since its operation started in 1989. The idea that beams collide only at the south IP where the BES detector is located to enhance luminosity has been investigated since 1991.[5] The basic scheme is to separate the electron and positron beams at north IP by 1 pair of vertical electrostatic separator. The early experiments did not give any positive results. The major reason is that the phase advance between separators is not exact $180^{\circ}$. The vertical orbit distortion created by north separators leak out to the active IP. In the fall of 1995 with adding 4 new power supplies for the insertion quadrupoles near north IP, a new lattice with eliminating one low- $\beta$ insertion was designed. The new configuration ensures the exact $180^{\circ}$ phase advance between two north separators. The horizontal and vertical $\beta$-functions at north IP become $4.62 \mathrm{~m}$ and $0.65 \mathrm{~m}$ respectively. The tunes of the ring, $v_{\mathrm{x}}=6.12$, above the integer, $v_{\mathrm{y}}=6.62$, above the half integer, are quite different from those of normal double-IP operation.

The single IP experiments are carried out since 1996 during machine studies. Up to now the best performance occurred with low- $\beta$ configuration in which the vertical $\beta$ function at south IP is $7 \mathrm{~cm}$. The beam-beam tune shift per revolution decrease significantly comparing with that in two IP's operation. The maximum beam current and beam-beam parameter have been increased, which results a higher luminosity than that with same $\beta$-function of two IP's operation. More experiments are going to be done to explore the potential with lower vertical $\beta$-function at south IP.

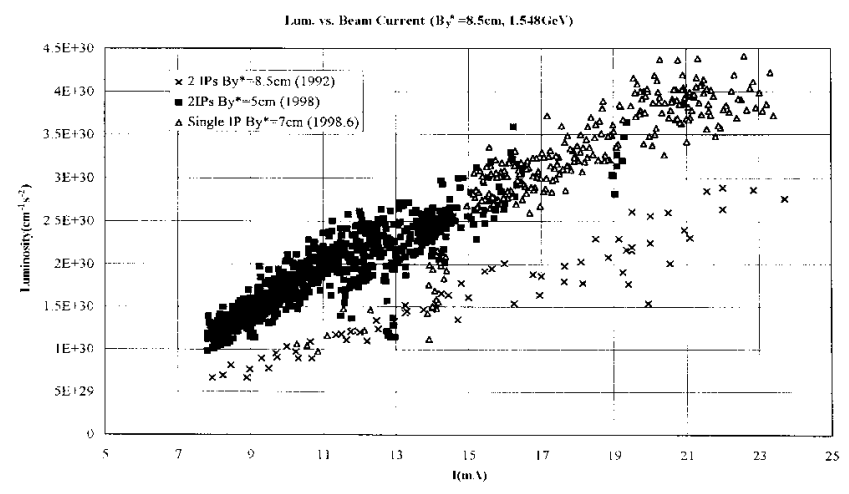

Figure 2: Luminosity increase with single IP

\subsection{Dedicated Synchrotron Radiation Operation}

BEPC is designed to serve as a research facility for both elementary particle physics and synchrotron radiation 
experiments. A low emittance configuration has been brought into routine dedicated synchrotron radiation operation mode since 1990 . The operating beam energy of this mode is $2.2 \mathrm{GeV}$ with natural horizontal emittance of $76 \mathrm{~nm} . \mathrm{rad}$, one order of magnitude smaller than that of collision mode for particle physics ( $660 \mathrm{~nm}$.rad at 2.015 $\mathrm{GeV})$. Recently a new lattice configuration aiming at operating machine at $2.5 \mathrm{GeV}$ by adjusting the focusing strengths of a few quadrupoles has been made. The natural horizontal emittance increases to $79 \mathrm{~nm}$.rad at 2.2 $\mathrm{GeV}$.

During the shutdown in 1996, a five-period permanent wiggler magnet was installed in the storage ring of BEPC in order to provide the higher performance photon beams. The period length is $30 \mathrm{~cm}$ and the peak magnetic field is 1.5 Tesla. The gap is adjustable from $3.9 \mathrm{~cm}$ to $22 \mathrm{~cm}$. Two new beamlines extracted from this wiggler magnet have been built. The higher flux photon beams provided by this new wiggler have attracted more users in various research fields from different universities and institutes. The beam current intensity in dedicated synchrotron operation is $80 \sim 100 \mathrm{~mA}$. The beam lifetime, which is dominated mainly by the Touschek effect, is increased to over 20 hours by filling 50-80 unequal bunches. The operation at higher beam energy, i.e., $2.5 \mathrm{GeV}$ is commissioned successfully. The parasitic operation mode with new wiggler in particle physics run is being tested.

\section{FUTURE UPGRADES}

The rich physics in $\tau$-charm energy region and the achievements in BEPC have been encouraging us to make continuous efforts in the further developments of collider facility.

The brightest option is to build a new research facility dedicated to the same energy region as BEPC but with highly superior performance as to luminosity (specifically $\left.10^{33} \mathrm{~cm}^{-2} \mathrm{~s}^{-1}\right)$ and incorporating additional parameters, such as the possibility of polarized circulating beams or specialised optics providing highly mono-chromatic beams, called $\tau$-charm Factory. The feasibility study has been going on since $1994[7,8,9]$. If this project is approved the existing BEPC storage ring will become a fully dedicated synchrotron radiation facility. A study to upgrade it to the higher energy $(3.0 \mathrm{GeV})$ and lower emittance $(<20 \mathrm{~nm} \cdot \mathrm{rad})$ is carried out[10].

On the other hand the possibility of continual improving the performance of BEPC is investigated. The major scheme of this improvement is multi-bunch operation by adopting pretzel scheme like CESR[11]. Figure 4 shows the schematic of this scheme on BEPC. Both horizontal and vertical pretzel schemes have been preliminarily exploited[12,13]. It seems that both separation schemes can meet the design requirements. The number of bunch, determined by the integer part of tunes of ring, can be 6 or 7 . The expected luminosity gain factor is $4 \sim 5$.

Besides the theoretical studies, some selected experiments

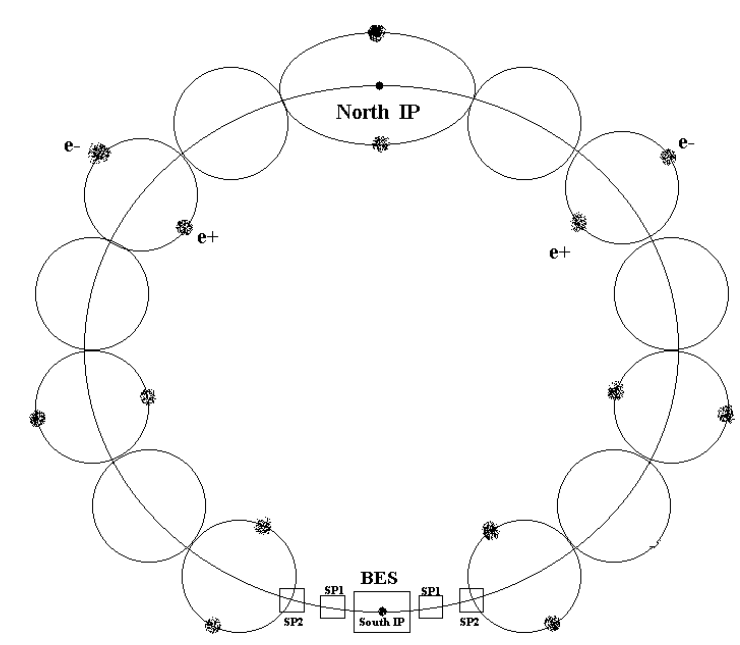

Figure 4: Pretzel Scheme with 7 bunches on BEPC

are performed during machine study time to investigate some key issues in future projects, such as multi-bunch instabilities especially the Photon-Electron Instability [14], horizontal and vertical pretzel orbits experiments with single beam, etc.

\section{SUMMARY}

BEPC is now operating with a nearly doubled luminosity after the upgrades in recent years. The plans are underway to greatly improve the luminosity in the future.

\section{ACKNOWLEDGMENTS}

This article is a brief review of the work done by many dedicated people in the accelerator and technical sector at IHEP. More detailed contributions on specific items can be found elsewhere in these proceedings.

\section{REFERENCES}

[1] S. X. Fang, S.Y. Chen, Proc. $14^{\text {th }}$ Int. Conf. On High Energy acc., 51(1989)

[2] Y. Z. Wu, PAC'91, San Francisco, 180(1991)

[3] C. Zhang, HEACC'92, Hamburg, 84(1992)

[4] Y. Z. Wu, BEPC Luminosity Upgrades Workshop, 184(1991)

[5] Z. Y. Guo et al., BEPC Performance Workshop'96, (1996)

[6] X. A. Luo et al., this proceeding(1999)

[7] Beijing Tau-charm Factory Feasibility Study Report(1996)

[8] N. Huang, et al., PAC'97, Vancouver (1997)

[9] D. Wang, Y. Z. Wu, ICFA Workshop on Beam Dynamics issues for e+e- Factories, Frascati, 397(1997)

[10] J.Q. Guo, L.M. Chen, IHEP/AC/AP/99-03(1999)

[11] D. Rice, PAC'93, San Francisco, 1978(1991)

[12] L.F. Wang, C. Zhang, PAC'97, Vancouver(1997)

[13] Q. Qin, BEPC Performance Workshop'98, 76(1998)

[14] Z.Y. Guo, et al., this proceeding(1999) 